OPEN ACCESS

Edited by:

Weida Tong,

National Center for Toxicological

Research (FDA), United States

Reviewed by:

Laura I. Furlong,

Mar Institute of Medical Research

(IMIM), Spain

Martin Romacker,

Roche, Switzerland

*Correspondence:

Thomas Luechtefeld tom@toxtrack.com

Specialty section:

This article was submitted to Medicine and Public Health,

a section of the journal

Frontiers in Artificial Intelligence

Received: 24 March 2021

Accepted: 13 July 2021

Published: 05 August 2021

Citation:

Bozada T, Borden J, Workman J,

Del Cid M, Malinowski J and

Luechtefeld T (2021) Sysrev: A FAIR

Platform for Data Curation and

Systematic Evidence Review.

Front. Artif. Intell. 4:685298.

doi: $10.3389 /$ frai.2021.685298

\section{Sysrev: A FAIR Platform for Data Curation and Systematic Evidence Review}

\author{
Thomas Bozada Jr${ }^{1}$, James Borden ${ }^{1}$, Jeffrey Workman ${ }^{1}$, Mardo Del Cid ${ }^{1}$, \\ Jennifer Malinowski ${ }^{2}$ and Thomas Luechtefeld ${ }^{1,3 *}$ \\ ${ }^{1}$ Insilica LLC, Bethesda, MD, United States, ${ }^{2}$ Independent Researcher, Hebron, CT, United States, ${ }^{3}$ Toxtrack LLC, Baltimore, \\ $M D$, United States
}

Well-curated datasets are essential to evidence based decision making and to the integration of artificial intelligence with human reasoning across disciplines. However, many sources of data remain siloed, unstructured, and/or unavailable for complementary and secondary research. Sysrev was developed to address these issues. First, Sysrev was built to aid in systematic evidence reviews (SER), where digital documents are evaluated according to a well defined process, and where Sysrev provides an easy to access, publicly available and free platform for collaborating in SER projects. Secondly, Sysrev addresses the issue of unstructured, siloed, and inaccessible data in the context of generalized data extraction, where human and machine learning algorithms are combined to extract insights and evidence for better decision making across disciplines. Sysrev uses FAIR - Findability, Accessibility, Interoperability, and Reuse of digital assets - as primary principles in design. Sysrev was developed primarily because of an observed need to reduce redundancy, reduce inefficient use of human time and increase the impact of evidence based decision making. This publication is an introduction to Sysrev as a novel technology, with an overview of the features, motivations and use cases of the tool.

Methods: Sysrev. com is a FAIR motivated web platform for data curation and SER. Sysrev allows users to create data curation projects called "sysrevs" wherein users upload documents, define review tasks, recruit reviewers, perform review tasks, and automate review tasks.

Conclusion: Sysrev is a web application designed to facilitate data curation and SERs. Thousands of publicly accessible Sysrev projects have been created, accommodating research in a wide variety of disciplines. Described use cases include data curation, managed reviews, and SERs.

Keywords: software, data extraction, evidence review, data management, machine learning, systematic review, meta analysis 


\section{INTRODUCTION}

A systematic evidence review (SER) is a methodological tool used in a variety of disciplines to capture all relevant published literature on a topic, which may lead to quantitative analysis of the data (meta-analysis) or qualitative synthesis. Core principles of SERs are rigor, reproducibility, and transparency. SERs generally follow a prescribed process, beginning with the identification of the population, intervention, comparator, and outcomes of interest. Once the relevant key questions have been developed, a search in one or more databases yields a number of articles that must be reviewed, typically in duplicate by blinded reviewers according to pre-specified inclusion and exclusion criteria. Data from included studies are abstracted into forms for subsequent quantitative analysis or qualitative synthesis.

One of the primary goals of Sysrev is to apply SER methods to other workflows, including generalized data curation, education, and any workflow involving collaboration between humans and between humans and machines for evaluation of digital documents.

Although SERs accompanied by meta-analysis are considered the highest level of evidence, due to their overall objectivity, they are time-consuming (Burns et al., 2011). Adhering to SER methodology can be difficult, particularly when the method is applied to non-biomedical research (Mallett et al., 2012). Thorough searches can require subscriptions to multiple databases. Depending on the topic, it is not unusual for a well-designed literature search to return more than 10,000 studies and for a SER to take more than 1 year to complete. To alleviate some of the inherent challenges with managing a large corpus of literature, the number of participants on a review may be quite large. Numerous software have been developed to help reviewers manage the literature base and perform one or more phases of a SER, such as citation management or title and abstract screening (van der Mierden et al., 2019). More recently, machine learning algorithms have been proposed as a way to increase workload efficiency (Gates et al., 2019). However, despite the plethora of available SER software, recent reviews have found few software can adeptly meet the needs of those who perform SERs across disciplines (Harrison et al., 2020).

While the number of SER publications has risen substantially in the past decade, much of the data extracted from their included articles remains locked away, limiting their reuse by others performing adjacent research. For example, data extraction templates may be published as a supplement to a SER, but the completed extraction forms for every study may not, or what is published is in a form that precludes secondary research. Therefore, data curation is an essential component of data reuse and research transparency/reproducibility.

There are a number of curated, publicly-available (open access) data repositories for scientific and academic disciplines, such as the worldwide Protein Data Bank (wwpdb.org), the Cambridge Structural Database (https://www.ccdc.cam.ac.uk/ solutions/csd-core/components/csd/), and the Publishing Network for Geoscientific and Environmental Data (PANGAEA) (https://www.pangaea.de/). However, re-use of the information can be limited due to controls on database access, limited labeling of semi-structured or unstructured data (Hariri et al., 2019), and the structures of databases that prevent inclusion of some experimental data (Wilkinson et al., 2016), among others. These obstacles contribute to "dark data" the data from small experiments which are unlikely to be openly shared with other researchers and exist only on controlled-access laboratory or university servers (Wallis et al., 2013). Consequently, much of publicly funded research remains relatively hidden and at risk for loss unless submitted to repositories, disproportionately affecting the "long tail" of science and technology (P. Bryan Heidorn, 2008).

The FAIR Guiding Principles were published to encourage better data stewardship and management and in support of infrastructure to minimize dark data (Wilkinson et al., 2016). The FAIR Principles refer to Findability, Accessibility, Interoperability, and Reuse of digital data. The findability tenet incorporates the assignment of unique identifiers and metadata to information so that it can be found more easily by humans or machines (especially search engines). Accessibility requires the data or metadata be retrievable using a standard communications protocol and that the metadata associated with the data remain available even after the original data is no longer accessible. Accessibility does not require all types of data be freely available to any user for any purpose; for example, the National Center for Biotechnology Information's dbGaP repository of phenotype and genomic information restricts access to datasets to NIH and senior investigators (Tryka et al., 2014). Incorporation of shared and standardized vocabularies are essential to support interoperability and reuse of data and its metadata. Importantly, FAIR Principles are discipline agnostic; that is, they can be applied across a range of academic and industrial activities. Since their 2016 publication, numerous disciplines are striving to apply FAIR Principles, including genomics, agriculture (Arnaud et al., 2020; David et al., 2020), climate science, and medicine.

Sysrev, which is in active development, is a FAIR motivated software to extract and label data from a variety of data sources. This paper describes the features behind Sysrev, provides use case examples, gives a brief meta-analysis of Sysrev open access data, and suggests future directions and enhancements to Sysrev.

\section{WORKFLOW}

The following section describes the user experience and project workflow within Sysrev.

First, Users access and create accounts at sysrev.com. All account types (including free accounts) can create an unlimited number of publicly accessible Sysrev projects. Public projects can be discovered by search engines like google and bing. All data generated in a public project can be discovered and downloaded on the internet.

Users with paid accounts can choose to designate any of their Sysrev projects to remain private. Private project data can only be accessed by project members. 


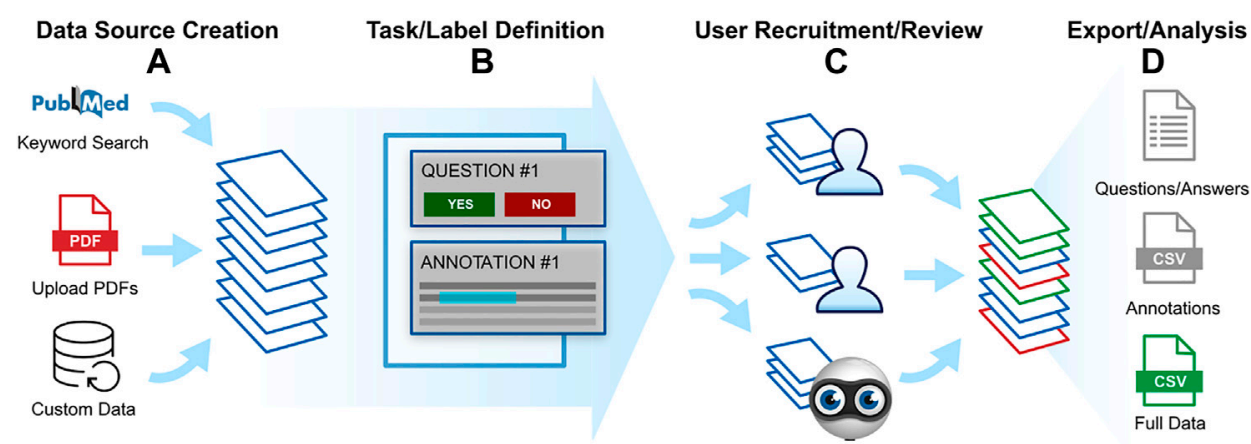

FIGURE 1 | Sysrev projects can be broken into 4 stages from left to right. (A) Articles are collected to form a data source. (B) Review tasks or "labels" are defined. (C) Reviewers are recruited and asked to complete review tasks. Active learning is involved at this stage to prioritize and replicate labelling tasks. (D) Data is exported and analyzed.

Sysrev projects can be broken down into 4 phases: data source creation, label definition, user recruitment/review, data export/ analysis and analysis (Figure 1).

\section{DATA SOURCE CREATION}

Sysrev projects are highly customizable and can accommodate a variety of data import structures. Currently supported file imports include XML, RIS citation format, and PDF. Text, JSON, and HTML formats are also supported via the Sysrev programming interface. A growing number of data integrations including pubmed.gov and clinicaltrials.gov searches.

\section{Future Development}

Sysrev is built to generalize the systematic review process to review any kind of digital document. Future sources will include support for images, videos, and other custom formats that can be viewed and annotated by users in a browser.

Future Sysrev sources will allow the use of one sysrev as a source for another sysrev, thus facilitating stagewise reviews and other applications involving review of reviews. Living reviews will be enabled by allowing sources to grow over time. Source publication will enable users to develop their own sources which can be shared between sysrevs. Sysrev already supports programmatic source publications at https://datasource.insilica. co, but these features are not yet integrated into the Sysrev user interface.

\section{TASK/LABEL DEFINITION}

Labels are structured data elements that are extracted from documents. Sysrev supports basic labels, group labels, and is developing many more advanced label types.

Sysrev supports three basic label types: Boolean, categorical and string labels. Label options allow labels to be set as "required," "requiring user consensus," as part of inclusion criteria, or set with a variety of other options.
"Required" labels must be provided by reviewers, reviewers cannot submit a document review until all required labels have been provided.

Labels that require user consensus will mark documents as "in conflict" when different reviewers disagree on the value associated with the label in a given document, this is a method for identifying documents with inconsistent user labels.

Labels with inclusion criteria rules are associated with document screening. Inclusion criteria options allow administrators to capture the reasons that a document was included or excluded.

Among a variety of other options, String labels can have associated regular expressions. Regular expressions allow users to write a pattern that must be met by user inputs. Regular expressions can be used to modify string labels to behave as numeric labels, date labels, dosage labels, or anything else that can be encoded in a regular expression, which is almost any pattern that can be written in text. Sysrev strongly recommends using regular expressions to normalize the string inputs that users can provide.

To create labels for a project users go to the manage -> label definitions section of a project.

Group labels allow project administrators to extract table data from documents. Group labels are composed of a set of basic labels. When extracting data into a group label, reviewers fill in rows in a table corresponding to the provided basic labels. Figure 2 provides a screenshot where a reviewer is extracting testing data from a safety data sheet (Bozada, 2020a). The ability to easily share review results on specific articles with a link lets users more easily work together. In this case users might share this result to discuss whether the "target" variable is correct, and point out that LC50 seems to be a distinct value from "eye" and "skin" in the "target" column. In this example, the project administrator wanted to know what test types were defined in the safety data sheet, and to associate related dose amounts, results, species, etc. Group labels are a powerful concept for creating relational databases from documents. When they are exported, each group label can be treated as a new table.

Label definitions are a generalizable concept. When they are built correctly, they communicate to reviewers specific 

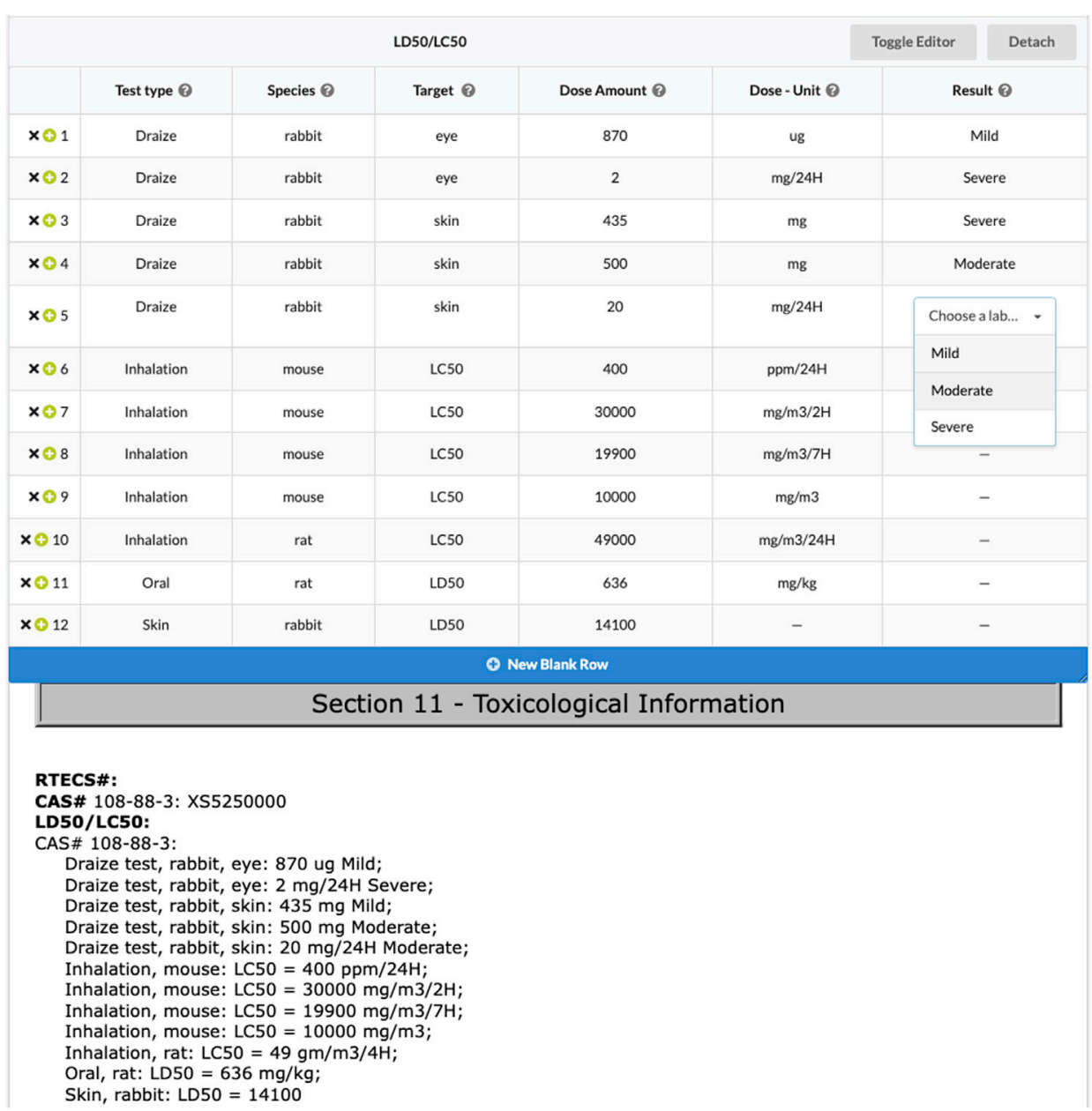

FIGURE 2 | Users extract testing information from a safety data sheet using a group label. The results of this safety data sheet review are available (with pdf hidden) at sysrev.com/o/2/p/31871/article/8296525.

constraints for extracting data from documents. They can even involve interactions with documents. For example, Sysrev supports "text annotation" which involves the selection of text within a document and the assignment of a value to the selection. Sysrev supports this advanced kind of text annotation label.

\section{Future Development}

Other advanced label types and label properties are in development, such as image annotation, shared labels which can be shared across many projects, and question/answer labels which will allow sysrev to function as an educational platform.

\section{USER RECRUITMENT AND REVIEW}

After creating document sources and defining labels, users must be recruited to begin the reviewing process. Many sysrev projects consist of a single project owner who also performs the full review. Other projects have hundreds of reviewers. Most fall somewhere in-between. Recruiting users can be done by sharing a link or sending emails directly from sysrev.com.

Sysrev also supports recruitment of existing Sysrev users using sysrev.com/users. Users can identify themselves as available for future reviews. Before inviting these users, project administrators can review previous user projects and performance by using sysrev.com/search or viewing the user profile page.

Users can be assigned to groups. Eventually these groups will allow project administrators to modify review prioritization and tasks for specific users. For example, reviewers could be grouped by language and assigned articles accordingly. Or, biology experts could be asked to perform one task, while chemistry experts are asked to perform a different task on the same documents.

User recruitment is one area where Sysrev is attempting to improve the visibility and community behind review work. Some Sysrev users have performed tens of thousands of document reviews, their experience and publicly visible work could make them strong candidates to help in other projects.

Document review is the heart of Sysrev. Sysrev distributes documents to reviewers and manages that distribution according 


\section{Label Counts}

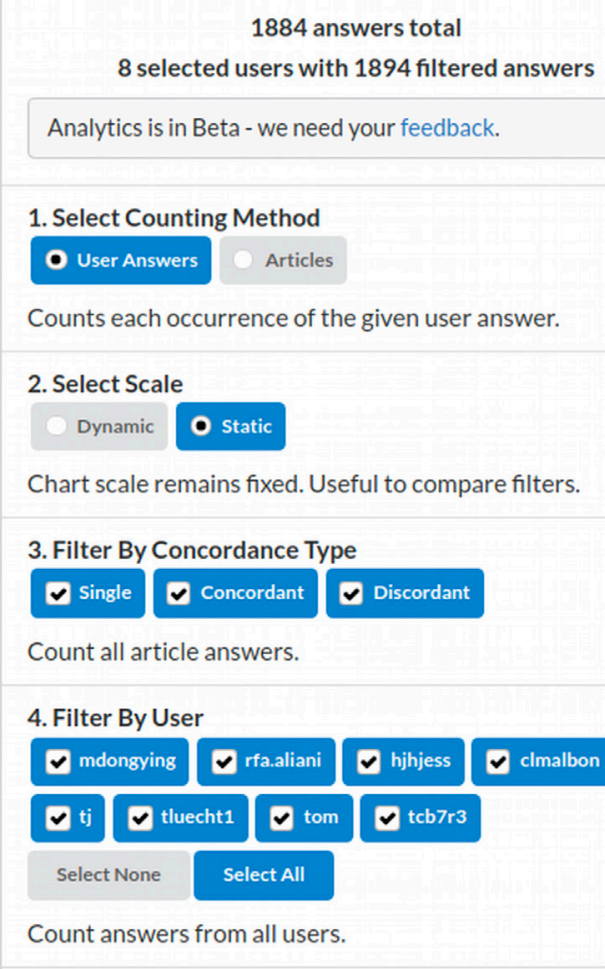

\section{Filter By Label Answer}

Count all articles regardless of answer content.

Click bar on chart to add filter.

6. Go To Articles $\rightarrow$

Open articles with label-answer and user filters.

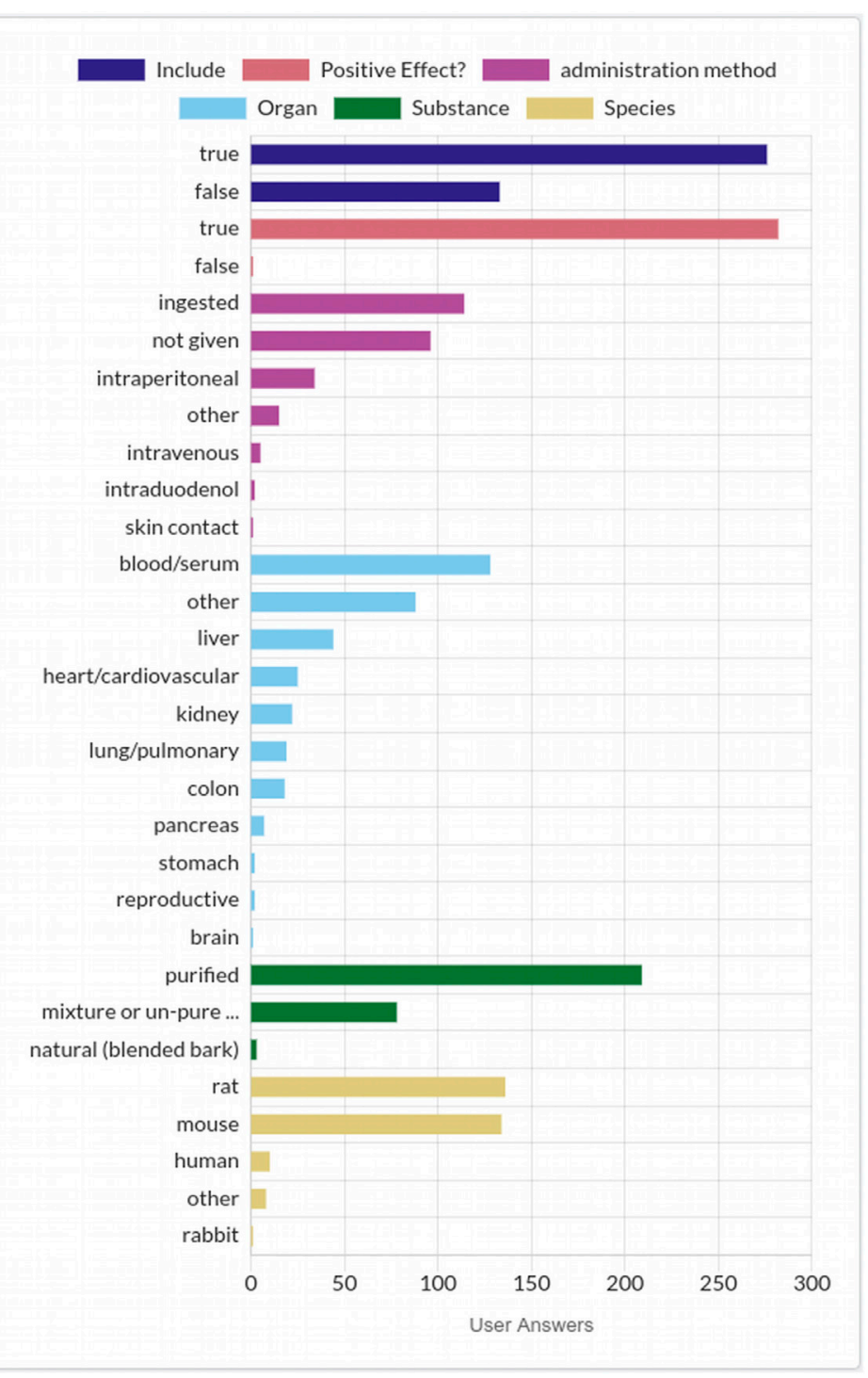

FIGURE 3 | A screenshot of the sysrev label counting tool. A demonstration of this tool is publicly available at https://sysrev.com/o/2/p/21696/analytics/labels. Label counts can be filtered, visualized and updated in real time as a sysrev progresses.

to several project parameters. Projects can require double review, single review, or unlimited review (where all reviewers review all documents). Review can be prioritized in order to maximize the rate of document review, in order to maximize the rate of review duplication, or to balance these priorities.

Unlimited review can be useful for classroom applications where students are evaluated according to some gold standard values associated with each document.

\section{Progression}

Project administrators can check in on their project at any time to keep track of completion and reviewer performance. Sysrev analytics features allow administrators to evaluate reviewer performance relative to other users. The distribution of extracted labels, and other informative analytics are provided on the project overview and analytics pages, and are updated in real time during review. Figure 3 shows the Sysrev label counting tool, which provides a visualization and filtering options to keep track of label distributions during project progression.

\section{Active Learning}

During the review process, Sysrev periodically creates machine learning models. These models attempt to replicate all binary and categorical review tasks. Models are also used to prioritize review, by selecting documents with the lowest classification confidence in an active learning paradigm (Settles, 2009). Anecdotally, when active learning was implemented on Sysrev, users reported that the more difficult documents in a review were quickly being prioritized. The motivation behind Sysrev's active learning is to maximize the value of human time. When machine learning 


\section{Step 1 - Label difficulty \\ Track label difficulty by comparing the number of articles where all $2+$ users agree (concordant) vs articles where $1+$ users disagree (discordant) \\ Only boolean labels with $1+$ double reviewed articles are shown. \\ Step 2 - User Performance \\ Discover which users have the best performance on the selected label. Select a label below, or click a bar in Step 1 \\ Select Label: \\ - Include Positive Effect?}
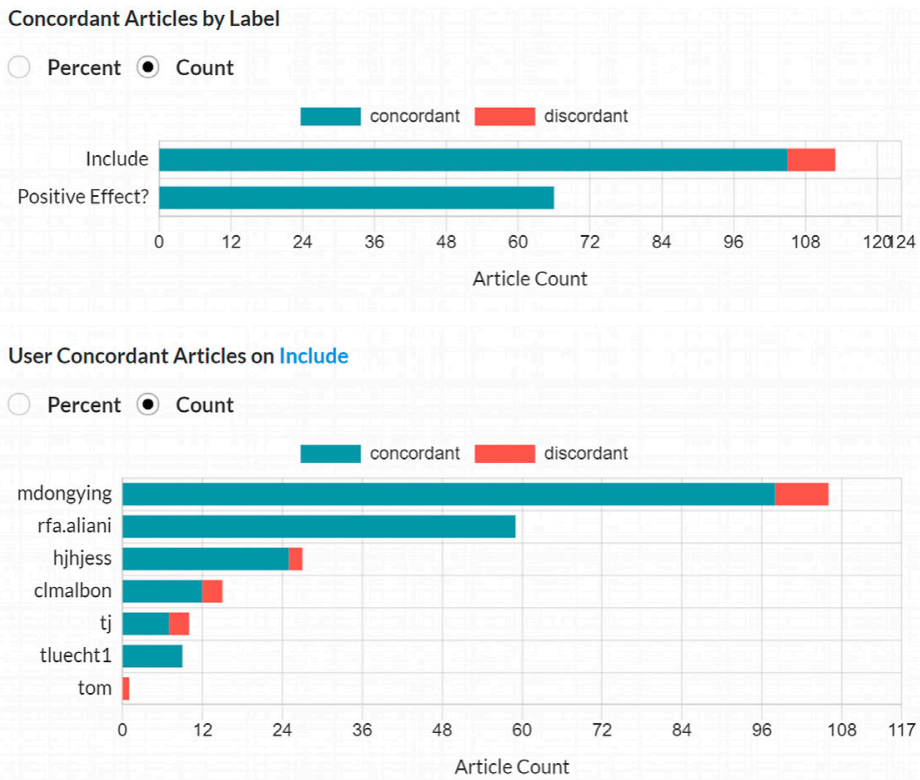

\section{Step 3 - User / User Investigation}

Select a label and a user below to discover user-user pairs are most concordant and discordant. Tracking concordance against your most trusted users can help discover difficult tasks or low performance reviewers.

Select Label:

- Include Positive Effect?

Select User:

o mdongying

rfa.aliani

hjhjess

clmalbon

tj tluecht1

tom
User Concordant Articles vs mdongying on Include

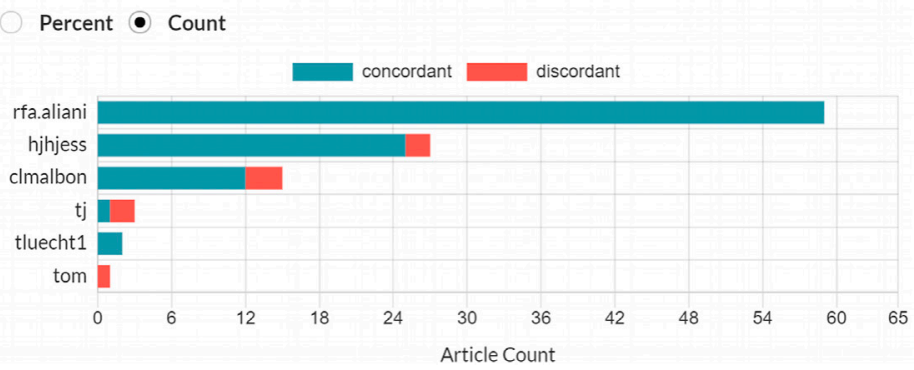

FIGURE 4 | A screenshot of the Sysrev concordance tool in action. This tool can be demonstrated at sysrev.com/p/21696/analytics/concordance.

models can accurately evaluate uncertainty, they can be used to prioritize human review to only those documents that are difficult to automate.

\section{Conflict Resolution}

A conflict flag is generated when users disagree about labels marked as "requiring concordance" in a specific document. Conflict flags identify documents where reviewers disagreed about one or more labels that require concordance. Once an article is flagged for conflict, the conflict can be resolved by a project administrator. By default, the inclusion label, which is used to screen articles in a sysrev, requires concordance.

\section{DATA EXPORT AND ANALYSIS}

Sysrev supports methods to export review data to spreadsheets, $\mathrm{xml}$ documents, and json files. In addition, a graphql API is available that allows users to programmatically access sysrev data. Two open source clients of this graphql API written in $\mathrm{R}$ and
Python are in development at github.com/sysrev/RSysrev and github.com/sysrev/PySysrev.

Sysrev provides a growing set of analytics tools built directly into each project.

\section{Concordance}

The Sysrev concordance tool allows project administrators to evaluate their labels and reviewers. Concordance is measured by counting how often users agree about extracted labels. Figure 4 demonstrates the concordance tool in a public review on mangiferin (https://sysrev.com/p/21696/analytics/concordance).

In the first step, administrators can quickly evaluate which tasks are most concordant. Highly concordant tasks are tasks that reviewers frequently agree on, and are more likely to be well defined and understood tasks. Highly discordant tasks may indicate areas where reviewers have been inadequately trained or tasks have been under developed.

In the second step administrators can view how concordant each user is on a given label. In Figure 4, User rfa.aliani has perfect concordance on the include label, and other users have varying levels of discordance. 


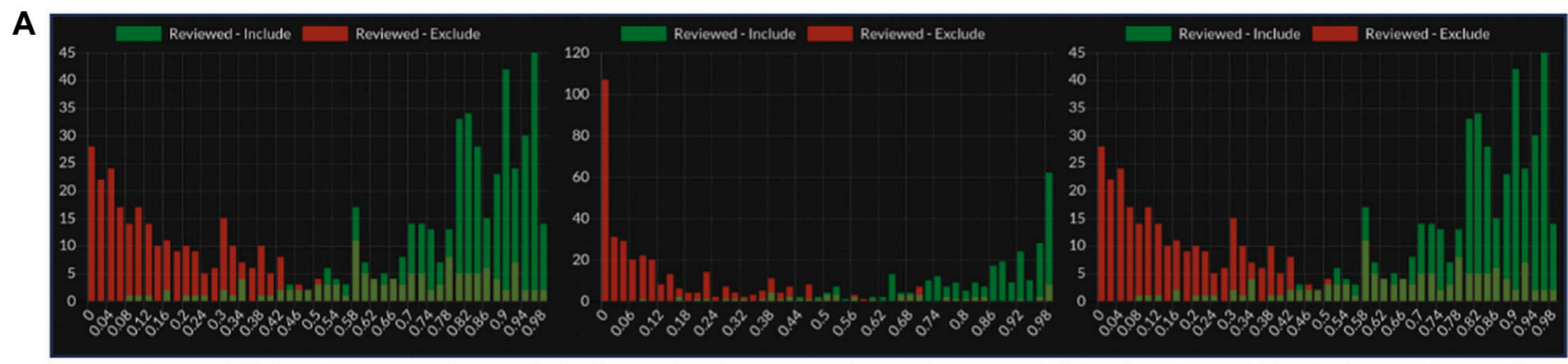

B

Cardioprotective effect of mangiferin on left ventricular remodeling in rats.

The purpose of this study was to clarify the protective role of mangiferin on postinfarction myocardial remodeling and potential mechanisms. The myocardial infarction (MI) model was established by ligating the left anterior descending coronary artery. Cardiac function, myocardial apoptosis and fibrosis, serum tumor necrosis factor- $\alpha$ (TNF- $\alpha$ ) and phosphorylated p38 mitogen-activated protein kinase (MAPK) were examined by echocardiography, histological staining, ELISA and Western blot, respectively. Mangiferin attenuated $\mathrm{MI}$ and prevented the development of intercellular fibrosis. Western blotting underscores that the p38 MAPK cascade plays an important role in the cardioprotective effect of mangiferin during MI. Inhibition of p38 MAPK significantly decreased serum TNF-a levels. Transferase-mediated uridine nick end labeling and Masson staining also showed that mangiferin reduced apoptosis and fibrosis in myocardium remodeling. Based on these results, we conclude that mangiferin has a therapeutic effect on post-MI left ventricular remodeling and improves cardiac function.

\begin{tabular}{|c|c|c|c|c|c|}
\hline \multicolumn{2}{|c|}{ Positive Effect? } & \multicolumn{2}{|l|}{ Substance } & \multicolumn{2}{|l|}{ Organ } \\
\hline Label & Prediction & Label & Prediction & Label & Prediction \\
\hline True & $76 \%$ & natural (blended & $23 \%$ & $\begin{array}{l}\text { heart/cardiovasc } \\
\text { ular }\end{array}$ & $91 \%$ \\
\hline \multirow[t]{4}{*}{ False } & $24 \%$ & & & & \\
\hline & & purified & $97 \%$ & blood/serum & $18 \%$ \\
\hline & & mixture or un- & $1 \%$ & reproductive & $22 \%$ \\
\hline & & pure extract & & liver & $26 \%$ \\
\hline
\end{tabular}

FIGURE 5 | (A) Machine learning prediction of article inclusion compared to actual reviewer inclusion decisions from 3 public sysrev projects on spinal surgery sysrev.com/p/14872, sysrev.com/p/14873, sysrev.com/p/14874. (B) Sysrev categorical and Boolean predictions. Sysrev predictions correctly identify that this article references a "positive effect" on "heart/cardiovascular." They provide a strong prediction of "purified" mangiferin substance, even though the article does not explicitly specify this. Model predictions can be seen on every article in a project. This screenshot comes from an Insilica.co project on Mangiferin (sysrev.com/p/24557/ article/7225450).

In the last step, administrators view users' concordance against a given user for a certain label. Figure 4 demonstrates that rfa.aliani has perfect concordance with mdongying for the include label, but that other users have some discordance with mdongying.

\section{Machine Learning}

Sysrev periodically builds machine learning algorithms that attempt to replicate all Boolean, categorical and (soon) annotation reviewer tasks. Sysrev provides some transparency around the accuracy for these models via a histogram describing model predictions for articles that human reviewers have already excluded and included. Machine learning models are updated periodically as articles are screened and new histograms are provided to evaluate model performance (Yang et al., 2009;
Luechtefeld and Bozada, 2019). Figure 5A provides several example histograms, from public Sysrev projects, which are automatically generated during project progression. These histograms show the number of articles included or excluded by human reviewers with a probability of inclusion given on the $x$ axis. Currently, Sysrev trains these models directly from the given projects text and user classifications, in the future Sysrev will use transfer learning to benefit projects using shared or similar labels.

Sysrev automatically builds models to replicate every categorical and Boolean label in every sysrev project. As of March 1, 2021 a total of 94,064,632 predictions have been made by Sysrev models for articles in sysrevs. The predictions for these labels can be viewed in articles and used to filter article lists. For example, after labeling a few hundred articles for a Boolean "heart/cardiovascular" label, the resulting model can be 
used to filter articles with a high probability of receiving the "heart/cardiovascular" label.

Figure 5B shows label predictions for 3 of the categorical/ Boolean labels for an article in a sysrev on Mangiferin, a mango extract. This review has many categorical and Boolean variables, three of which are:

Positive effect-Is the discussed effect a positive or negative one?

Substance-What form of mangiferin was used? Natural, purified or mixture.

Organ-What organ(s) were referenced: Heart, blood, reproductive, liver, etc.

Sysrev models provide a value between 0 and 1 (interpreted as a percentage) for each label + answer pair. Boolean labels receive two predictions, one for label + true and one for label + false. Categorical labels receive a prediction for each label + answer, these values do not necessarily sum to 1 , although future features will update model predictions to normalized values for single answer (mutually exclusive) categoricals.

\section{SUPPLEMENTAL FEATURES}

Sysrev develops a growing set of features that are supplementary to the core workflow features.

\section{Payment Platform}

Sysrev provides a payment platform where project administrators can deposit and distribute funds to reviewers on a per-document reviewed basis. In the future, Sysrev's payment platform will integrate modeling, previous user performance, and the quality/ difficulty of document reviews into rewards. We hope to provide a marketplace to link data curation projects to reviewers.

\section{Project Cloning}

One unique aspect of Sysrev is the ability to clone projects. This provides researchers working on related sysrevs, others who want to use the same data templates, or those who want to work with shared data, an opportunity to streamline project setup and ensure consistency in how data is extracted/curated across projects. Cloned sysrevs maintains the same labels and structure as the initial review, ensuring reproducibility. One example of a cloned project is a SER of methods used to quantify the mechanical work of breathing during exercise (available at: sysrev.com/p/27698). After the initial review was completed, the authors cloned the existing project to update the literature search (see sysrev.com/p/50226). Project cloning is motivated by analogy between sysrev projects and git repositories, we aim to make sysrev projects easier to copy and facilitate the dissemination of review protocols.

These are the major features behind a Sysrev workflow. The Sysrev development roadmap is driven by user requests. Please contact us at info@sysrev.com with any requests.

\section{Natural Language Processing Tools}

In addition to the above referenced machine learning tools, Sysrev integrates several natural language processing tools. Most notably, important terms are extracted from articles via a TF-IDF method and displayed on project overview pages. Significant text processing is involved in the machine learning and NLP steps on Sysrev. Text annotation is also possible and will soon integrate into a basic named entity recognition tool, this process already works for developers (see blog. sysrev.com/simple-ner).

\section{TUTORIALS}

Sysrev provides documentation of new features and tutorials in it's blog (blog.sysrev.com). Video tutorials and user discussions are given on the Sysrev youtube channel.

\section{FAIR MATURITY}

Sysrev aims to be a FAIR conforming web application. Currently, this is achieved through the availability of public projects on Sysrev. Users can make their projects easily discoverable by search engines like Google and Sysrev's internal search engine. Data in public projects can be downloaded by anybody. Sysrev's R integration, RSysrev, makes it possible to programmatically access data within projects owned by a user. Soon, RSysrev will allow users to programmatically access all public projects. Sysrev also offers shared labels and project clonability wherein labels defined for one project can be reused in another project, or an entire project structure can be copied to form the skeleton for a new project. While these features make Sysrev an excellent platform for sharing data curation projects, Sysrev still cannot be called a fully FAIR platform.

Over time, Sysrev will continue improving it's FAIR conformance across each domain-Findability, Accessibility, Interoperability and Reusability. A strong description of these guiding principles is provided by the FAIR Toolkit (Wise et al., 2019; Plasterer et al., 2021).

\section{APPLICATIONS}

As an easily accessible, web-based platform, Sysrev is able to serve a variety of functions across many fields. As a non-exhaustive list, Sysrev has been used for SERs, scoping reviews, evidence and gap-maps, meta-analyses, data curation, and annotation and has public projects in the fields of medicine, public health, psychology, chemistry, toxicology, ecology, geology, agriculture, political science, economics, and education. Sysrev has also been used as an educational tool to teach the underlying methodologies of literature review and standardized data curation. 


\section{CASE STUDIES}

As an exhaustive list of applications is impractical for this publication, we instead invited early Sysrev users to voluntarily describe their own projects. These case studies serve as a demonstration of several possible use cases for Sysrev. The case studies have been abbreviated here for space; the full versions are available as a Supplementary Files as Data Sheet 1.

\section{Ascorbate Administration Methods in Oncology Clinical Trials (Paller et al., 2020)} Author: Channing J Paller ${ }^{1}$, Tami Tamashiro' ${ }^{2}$, Thomas Luechtefeld $^{3}$, Amy Gravell ${ }^{2}$, Mark Levine ${ }^{4}$

1. Johns Hopkins School of Medicine

2. EMMES

3. Insilica LLC

4. National Institute of Health/National Institute of Diabetes and Digestive and Kidney Diseases.

Sysrev: https://sysrev.com/p/6737-Vitamin C Cancer Trials.

Description A review was performed of all clinical trial data from trials submitted to clinicaltrials.gov matching condition "cancer" and intervention "ascorbic acid," "ascorbate," or "vitamin c." Information regarding administration methods (dosing, oral vs. IV, multivitamin delivery), trial parameters (blinding, placebo, and randomization methods), drug combinations and disease types was extracted and organized for analysis. Analysis identified increased use of ascorbate alone and in combination with other therapies across multiple diseases. A high resolution evaluation of these outcomes is available as a book chapter in "Vitamin C and Cancer-An Overview of Recent Clinical Trials" (Paller et al., 2020).

\section{Data Curation of Chemical Safety Data Sheets}

Author: Daniel Mcgee, Wendy Schlett and Brad Van Valkenburg at Foresight Management.

Sysrev https://sysrev.com/p/4047 many private reviews.

\section{Description}

Safety Data Sheets (SDS) are documents containing the properties and occupational health, environmental hazards, and safety information for chemicals. SDS follow a consistent framework to align with the United Nations' Globally Harmonized System of Classification and Labelling of Chemicals (GHS) and are required to be available to downstream users, who may include manufacturers or transporters. Chemicals of concern vary according to the purpose and for each company. For example, the European Chemicals Agency restricts the manufacturing, marketing, or use of substances under the Registration, Evaluation, Authorization, and Restriction of Chemicals (REACH) Regulation while the California Office of Environmental Health Hazard Assessment maintains a list of chemicals known to cause cancer, birth defects, or other reproductive harm (Proposition 65) (Kilgore, 1990).

Sysrev was used by the Sustainable Research Group (SRG, now Foresight Management) to automate the extraction of relevant information from SDS to build a chemical of concern database for SRG clients. In addition to data from the SDS, SRG could label data with the name of the downstream user (account) or product, enabling cross-referencing to other chemical databases. This process facilitated attainment of LEVEL certification, an environmental certification for furniture similar to LEED certification for buildings, for a client (Luechtefeld, 2019).

\section{Methods Used to Quantify the Mechanical Work of Breathing During Exercise Author: Troy J Cross ${ }^{1}$, JW Duke ${ }^{2}$}

1. Mayo Clinic and The University of Sydney, Australia

2. Northern Arizona University, United States

\section{Sysrevs.}

https://sysrev.com/p/24827 and https://sysrev.com/p/ 46939-Title and Abstract screening.

https://sysrev.com/p/25285 and https://sysrev.com/p/ 49357-Full-text screening.

https://sysrev.com/p/27698 and https://sysrev.com/p/ 50226-Data extraction.

\section{Description}

The overarching purpose of this project is to survey the available literature for the many different methods used to quantify the mechanical work of breathing ( $\mathrm{Wb}$ ) during exercise. Each method of quantifying $\mathrm{Wb}$ has its own advantages and disadvantages, and their own technical requirements. Given the variety of methods used to measure $\mathrm{Wb}$, we thought it would be prudent to systematically evaluate the literature and determine the relative popularity of each method, and to assess the quality of their implementation. By conducting this systematic review, we hope to raise awareness of the advantages/pitfalls associated with each method of quantifying $\mathrm{Wb}$, and to emphasize the importance of reproducibility through clear descriptions of such methods. In doing so, we anticipate that these methods of quantifying $\mathrm{Wb}$ during exercise become more accessible to other Investigators in the area wishing to delve into the field of applied respiratory mechanics.

\section{Systematic Evidence Reviews: COVID-19 and Kidney Transplantation}

Author: Ciara Keenan at Campbell United Kingdom and Ireland, Queen's University, Belfast.

\section{Sysrevs.}

https://sysrev.com/p/29506-COVID19 CKD. https://sysrev.com/p/30488-COVID19 CKD ROBINS-I. 


\section{Description}

Numerous SERs have been performed using the Sysrev platform for one or more of the SER phases (screening, full-text review, and/or data extraction). An international team of researchers performed a SER using Sysrev to investigate the risks of COVID-19 to kidney transplant recipients (Bozada, 2020b; Mahalingasivam et al., 2021). The reviewers assessed both English- and Chineselanguage articles, uploading the studies as two separate xml files. Sysrev allows users to sort results based on the import file; the team of reviewers used this function to review only the abstracts in their primary language.

A separate Sysrev project was created to manage the data extraction phase of the SER. As with article screening, the reviewers were able to sort the included studies by import file. The extraction form consisted of twenty-nine questions consisting mainly of string and categorical labels (e.g., age, health status). For the 31 included articles, more than 700 pieces of information were extracted, including the risk of bias for each study.

A third Sysrev project was created for the team to determine the risk of bias for each included study in the SER (available at: https://sysrev.com/u/249/p/30488). The Risk of Bias in Non-randomized Studies of Interventions (ROBINS-I) assesses risk of bias that may occur preintervention, at intervention, and post-intervention for a study. Domains evaluated include confounding, selection of participants into the study, classification of interventions, deviations from the intended interventions, missing data, measurement of outcomes, and selection of reported results. Each domain is individually examined, and an overall risk of bias is determined (Sterne et al., 2016). The authors created categorical labels for each of the ROBINS-I domains and an overall risk of bias.

\section{Wildlife Trade and Human Disease}

Author: Hernan Caceres-Escobar ${ }^{1}$, Ciara Keenan ${ }^{2}$, Jon Paul Rodriguez $^{3}$, Richard Kock ${ }^{1}$

1. Royal Veterinary College, University of London.

2. Campbell United Kingdom and Ireland, Queen's University, Belfast.

3. IUCN Species Survival Commission.

Sysrev: https://sysrev.com/p/43801 - Wildlife Trade.

\section{Description}

The COVID-19 pandemic caused by SARS-CoV-2 is the latest in a series of novel diseases to affect humanity over recent decades. This has been counter to the trend of reduction in infectious disease burden enjoyed by humanity over the 20th Century due to the explosion in medical technologies and preventive measures. Initial circumstantial evidence suggested SARS-CoV-2 might have spilled into humans in a live wildlife trade market (commonly known as "wet markets") in the city of Wuhan (Hubei Province, China), where multiple domestic and wildlife species and products were processed and sold.
This is not the first time human infectious diseases have been associated to the diverse trade in wildlife species, some proven such as the 2003 monkeypox outbreak (United States), others unproven such as the 2002-2004 SARS outbreak (China) and COVID-19 index cases associated with Wuhan Market, China. Other human cases such as Ebola Virus infection were traced on a few occasions to index patients in contact with dead primates, great apes, and other wildlife species hunted and traded for food. The origins of HIV/AIDS are thought to be from evolution of simian (primate) origin virus (SIV) and the final example is during the bird flu pandemic (H5N1) in 2006, where a small number of imported birds into Europe showed infection with this virus, which led to a ban, having identified this wildlife trade hazard. Overall, zoonosis from wildlife trade appears to be rare (low risk or underreported), but as the examples illustrate, on occasions are of considerable public health concern. Given the seriousness of emerging pathogens, which have correctly or incorrectly been associated with wildlife trade, multiple organisations are now calling for bans on wildlife trade (i.e., from blanket bans to more nuanced and species-specific regulations). Wildlife trade has, despite the lack of hard evidence, been singled out as one of the main drivers of novel human disease emergence (UNEP, ILRI, 2020; Daszak et al., 2020). In truth, the role of the wildlife trade in these events and zoonosis risks other than those mentioned above, remain poorly understood and largely undocumented.

In this project (Cáceres-Escobar et al., 2020), we will identify and map all existing research evidence that primarily focuses on potential links between wildlife trade and human diseases (emerging human pathogens and zoonoses) and secondary elements that may be of importance to the development of evidence-based responses will also be included. Using robust search, retrieval, and methodological approaches to minimize potential sources of research bias with the proposed Evidence and Gap Map (EGM), we will summarize the existing and emerging evidence for the first time. The map will be made publicly available and interested parties will have access to a visual presentation of knowledge of the role of wildlife trade on human infectious diseases.

\section{Education: Coursera Evidence-Based Toxicology}

Author: Lena Smirnova at Center for Alternatives to Animal Testing at Johns Hopkins University, Bloomberg School of Public Health.

\section{Sysrev}

https://sysrev.com/p/3509-Evidence-Based Toxicology Assignment 2018.

https://sysrev.com/p/27474-EBT Course Assignment Year 3 https://sysrev.com/p/29023-GU systematic review $2019 / 2020$.

\section{Description}

Sysrev has value for educational purposes as a way to train individuals in SER practices. Dr. Lena Smirnova created an Evidence-Based Toxicology program on Coursera with a sysrev 
based assignment. Students were asked to apply SER principles in a final assessment that requires them to screen studies and resolve conflicts with other students according to pre-specified inclusion and exclusion criteria (available at: sysrev.com/p/3509). Over 411 students have now completed the course and thousands of labels have been assigned in the sysrevs. These courses indicate the potential for Sysrev to be used in teaching while simultaneously organizing valuable data from thousands of documents. The ease with which reviewers can navigate Sysrev and its alignment to FAIR principles make it an ideal choice for educational purposes.

\section{Limited Understanding of Bushfire Impacts on Australian Invertebrates (Saunders et al., 2021)}

Author: Manu E. Saunders ${ }^{1}$, Philip S. Barton ${ }^{1,2}$, James R. M. Bickerstaff $^{3}$, Lindsey Frost ${ }^{1}$, Tanya Latty ${ }^{4}$, Bryan D. Lessard ${ }^{5}$, Elizabeth C. Lowe ${ }^{6}$, Juanita Rodriguez ${ }^{5}$, Thomas E. White ${ }^{4}$, Kate D. L. Umbers ${ }^{3,7}$

1. School of Environmental and Rural Sciences, University of New England, Armidale, NSW, Australia.

2. School of Science, Psychology and Sport, Federation University Australia, Mount Helen, VC, Australia.

3. Hawkesbury Institute for the Environment, Western Sydney University, Penrith, NSW, Australia.

4. School of Life and Environmental Sciences, University of Sydney, Sydney, NSW, Australia.

5. Australian National Insect Collection, CSIRO, Canberra, ACT, Australia.

6. Department of Biological Sciences, Macquarie University, Sydney, NSW, Australia.

7. School of Science, Western Sydney University, Penrith, NSW, Australia.

Sysrev: https://sysrev.com/p/24557- Fire and Australian invertebrates.

\section{Description}

To understand existing knowledge, we synthesized the published literature using a standardized review method to find out what evidence is available to inform invertebrate conservation policies in the age of extreme fire events. We used a structured search in Scopus to collate peer-reviewed studies that measured the impacts of fire on any group of invertebrates in an Australian ecosystem. We then used Sysrev to screen studies via abstracts, before collating the final set of studies for manual data extraction.

Our results show that there is very little awareness of how fire affects invertebrate communities in Australian ecosystems. Peer reviewed studies were available for only six of the more than 30 invertebrate phyla and $88 \%$ of targeted studies were on arthropods, predominantly ants. Nearly all studies (94\%) were conducted on land, with only four studies measuring impacts in freshwater habitats. We found no studies of impacts on marine invertebrates in Australian waters, which is concerning-ash and post-fire runoff can have major effects on marine ecosystems, yet we didn't find any evidence of how this is affecting marine invertebrates. This is a real problem because fire risk and severity is increasing globally. For effective climate change and disaster recovery policies, we need to know how to identify key risks and opportunities for ecosystem recovery and biodiversity conservation in the face of these new challenging conditions.

\section{Pre-Clinical and Translational Studies on Macrophage Polarization in Nanoparticle-Based Cancer Immunotherapy}

Author: Colette Bilynsky, Wonhee Han, Anushree Gupta, Dasia Aldarondo, Hannah Fox, Chloe Brown, Archippe Mbembo, Megha Anand, Marissa McAfee, Jacob Bauldock, Melanie Gainey, Sarah Young and Elizabeth Wayne at Carnegie Mellon University.

\section{Sysrev}

https://sysrev.com/p/31994-Phase 1 title/abstract screening. https://sysrev.com/p/37476-Phase 2 title/abstract. https://sysrev.com/p/43536-Full text screening.

\section{Description}

The protocol for this project is registered on the Open Science Framework (Bilynsky et al., 2021).

The recent recognition of cancer as an inherently immunological disease has led to interesting opportunities for immunotherapy development. Tumors thrive by promoting an immunosuppressive environment, up-regulating non-malignant stromal cells that support tumor proliferation and migration and down-regulating those that might support a cytotoxic immune response. Tumor-associated macrophages (TAMs) are an important component of the tumor microenvironment, comprising up to $50 \%$ of the cells in solid tumors (Zhou et al., 2020). When TAMs are activated towards M2 polarization, which is anti-inflammatory and pro-tumoral, they contribute to tumor progression and indicate worse patient survival. Many therapy strategies have attempted to alter the polarization of macrophages' "re-programming" or prevent monocyte tumor infiltration (Noy and Pollard, 2014).

Nanoparticles are widely used to enhance the ability to target macrophages within tumor environments. Nanoparticles enable the customization of particle chemistries to modulate body pharmacokinetics, the encapsulation of multiple drugs or drugs that are water insoluble, and allows tunable control of drug release rates. However, nanoparticle customizability creates an exponential number of potential therapy design strategies. Thousands of studies have been published on the application of nanotechnology in cancer treatment and diagnosis but there have only been a very small number of FDA approved nanotherapeutics (Salvioni et al., 2019). This translational gap suggests that there is much to understand between nanoformulations of cancer therapeutics that are falling short of clinical implementation.

The purpose of this scoping review is to utilize data taken from studies implementing nanomedicine for cancer treatment and 
diagnosis and determine how these formulations modulate tumor-associated macrophage polarization. Specifically, we seek to answer whether there is an identifiable relationship between nanoparticle characterization (i.e., type, size, shape charge, cargo) and their ability to modulate macrophage polarization in the tumor microenvironment. This will allow us to assess the field of cancer therapeutics to understand the effectiveness of nano-therapeutics in an immunological context. This work is unique in its field as a comprehensive, systematic scoping review of how cancer nano-therapeutics interact with macrophages. Some previously published systematic reviews in cancer nano-medicine have focused on specific subsections, like using nanoparticles for actively-targeted delivery of chemotherapeutics or herbal medicine (Muhamad et al., 2018). Meanwhile, systematic reviews in cancer research have recently increased in frequency. However, these reviews mostly focus on clinical data with patients and not on research using in vitro or animal models of cancer (Kelley and Kelley, 2018). Thus, our scoping review has a role in the field as a systematic overview of cancer nano-therapeutics to provide understanding of how these formulations affect drug development and efficacy in treating cancer.

Sysrev has proven to be an excellent platform for managing the screening of thousands of studies by a large collaborative team new to the evidence synthesis process. Moreover, we have taken advantage of Sysrev's machine learning capabilities to automatically exclude a large number of articles using the predictions made based on the manual inclusion and exclusion of studies. This has greatly reduced the time and effort required to get through the title/abstract screening phase of the project allowing us to proceed more quickly to full text screening and data extraction.

\section{Indigenous Knowledge on Climate Change Adaptation: A Global Evidence Map of Academic Literature}

Author: Jan Petzold ${ }^{1}$, Nadine Andrews ${ }^{2}$, James D. Ford ${ }^{3}$, Christopher Hedemann ${ }^{4}$, Julio C. Postigo ${ }^{4}$

1. Center for Earth System Research and Sustainability, University of Hamburg, Hamburg, Germany

2. The Pentland Centre for Sustainability in Business, Lancaster University, Lancaster, United Kingdom

3. Priestley International Centre for Climate, University of Leeds, Leeds, United Kingdom

4. Department of Geography, Indiana University Bloomington, Bloomington, United States.

Sysrev: private project.

\section{Description}

We conducted a scoping review of peer-reviewed academic literature to support better integration of the existing and emerging research on indigenous knowledge in IPCC assessments. The research question underpinning this scoping review is: How is evidence of indigenous knowledge on climate change adaptation geographically and thematically distributed in the peer-reviewed academic literature?

As the first systematic global evidence map of indigenous knowledge in the climate adaptation literature, the study provides an overview of the evidence of indigenous knowledge for adaptation across regions and categorizes relevant concepts related to indigenous knowledge and their contexts in the climate change literature across disciplines. The results show knowledge clusters around tropical rural areas, subtropics, drylands, and adaptation through planning and practice and behavioural measures. Knowledge gaps include research in northern and central Africa, northern Asia, South America, Australia, urban areas, and adaptation through capacity building, as well as institutional and psychological adaptation. This review supports the assessment of indigenous knowledge in the IPCC AR6 and also provides a basis for follow-up research, e.g., bibliometric analysis, primary research of underrepresented regions, and review of gray literature.

\section{Synthesized Evidence on Conflict and War: A Systematic Map and Critical Appraisal of Systematic Reviews}

Author: Zahra Saad ${ }^{1}$, Tamara Lotfi $^{1}$, Hussein Ismail ${ }^{2}$, Neal Haddaway ${ }^{3}$, Elie $\mathrm{Akl}^{1}$

1. The Global Evidence Synthesis Initiative (GESI) Secretariat, American University of Beirut (AUB), Lebanon

2. American University of Beirut, Lebanon

3. Stockholm Environment Institute, Stockholm.

\section{Sysrev}

https://sysrev.com/p/39400-Example of critical appraisal. https://sysrev.com/p/36852-Example of data abstraction.

\section{Description}

The Global Evidence Synthesis Initiative (GESI) Secretariat is developing an evidence gap map of published evidence synthesis in the humanitarian field. The objective of the systematic mapping is to:

1) rigorously identify and critically appraise published systematic reviews in the humanitarian field, specifically on conflict and war.

2) publish a user-friendly, interactive and readily digestible map of the evidence accessible to stakeholders including humanitarian practitioners, policy makers, and researchers.

The team developed search strategies for 11 databases, and then completed title and abstract screening for 3,214 records. The full text screening of 601 eligible papers led to the inclusion of 340 evidence synthesis products. Then, the GESI Secretariat invited the GESI Network members to take part in data abstraction and critical appraisal as a collaborative approach using the free online software Sysrev. 
The GESI Secretariat connected with the Sysrev team and presented an introductory webinar to train the participants on how to use the platform. To split the work, the participants were divided into groups of 2 reviewers each. On Sysrev, 12 cloned data abstraction projects of twenty-four variables were created and eight cloned critical appraisal projects of 11 variables were formed, example links for access to these public projects are given above. The software offered a feature to view discrepancies in the answers allowing reviewers working in pairs to discuss them and accordingly resolve disagreements. At a later stage, the admin of the project exported all data in an excel format and then combined all results for data analysis. The team is currently developing the manuscript.

\section{EntoGEM: A Community-Driven Systematic Mapping Project to Build a Global Evidence Map of Insect Population and Biodiversity Trends}

Author: Eliza M. Grames ${ }^{1}$, Graham A. Montgomery ${ }^{2}$

1. University of Connecticut, Storrs United States

2. University of California Los Angeles, LA United States

Sysrev: https://sysrev.com/p/16612-EntoGEM: a systematic map of global insect population and biodiversity trends.

\section{Description}

EntoGEM is a community-driven project that aims to compile evidence about the current status and trends of global insect populations and biodiversity. Our goal is to build a systematic map database that contains all studies and datasets, published and unpublished, that contain evidence regarding long-term insect population and biodiversity declines, increases, or lack of changes over time. The final product will be publicly accessible, interactive, and searchable by metadata coded for each study, such as location, taxa, duration and time period of study, and other variables listed in the pre-registered protocol (Grames et al., 2019). By identifying what studies and datasets exist, this project will help prioritize future research on insect conservation, guide granting by funding agencies, and facilitate follow-up syntheses.

Because the comprehensive search for the project yielded over 144,000 results, we are using SysRev to complete targeted subsets of the search results, which then feed back into topic models that help to prioritize future screening efforts by the community. Although only roughly $5 \%$ of the EntoGEM search results have been screened by the community so far, already we have identified more than one hundred datasets that contain 10 or more years of insect population and biodiversity data that have not been referenced in any reviews, meta-analyses, or syntheses on insect decline to date (Grames, 2020).

\section{Funding}

EntoGEM was the first winner of the Sysrev mini-grants, small grants to incentivize public health research. The project is also funded by the Doris Duke Charitable Foundation.

\section{Systematic Evaluation of the Application of Zebrafish in Toxicology}

Author: Kristin Ryan ${ }^{1}$, Jon Hamm ${ }^{2}$, Neepa Choksi ${ }^{2}$, Lauren Browning $^{2}$ and Nicole Kleinstreuer ${ }^{3}$

1. National Institutes of Health/National Institute of Environmental Health Sciences/Division of the National Toxicology Program/Systems Toxicology

2. Integrated Laboratory Systems, LLC.

3. National Institutes of Health/National Institute of Environmental Health Sciences/Division of the National Toxicology Program/National Toxicology Program Interagency Center for the Evaluation of Alternative Toxicological Methods.

\section{Sysrev \\ https://sysrev.com/p/54429-Amoxicillin Part 1. https://sysrev.com/p/55474-Aspirin Part 1. \\ 22 other related reviews.}

\section{Description}

NICEATM is currently working on a project entitled Systematic Evaluation of the Application of Zebrafish in Toxicology (SEAZIT), aimed at understanding the utility of zebrafish for toxicological screening and the impact of key protocol variations on the data generated by this small model organism system. The current phase of SEAZIT involves an interlaboratory study utilizing 39 chemicals to compare several exposure conditions. Developmental toxicity results and outcomes available in published literature on these chemicals will be used to interpret the outcome of the interlaboratory study. Broad literature searches focused on retrieval of developmental toxicity studies in mammalian and zebrafish models have been conducted in PubMed. Relevant studies (5-10 per chemical) will be reviewed and used to assist in results interpretation from the proposed interlaboratory study.

\section{Funding}

This project was funded with federal funds from the NIEHS, NIH under Contract No. HHSN273201500010C.

\section{Cardiotoxicity Evaluation}

Author: Nicole Kleinstreuer ${ }^{1}$, Neepa Choksi ${ }^{2}$, Lauren Browning ${ }^{2}$, Amber Daniel ${ }^{2}$

1. National Institutes of Health/National Institute of Environmental Health Sciences/Division of the National Toxicology Program/National Toxicology Program Interagency Center for the Evaluation of Alternative Toxicological Methods

2. Integrated Laboratory Systems, LLC.

\section{Sysrev}

https://sysrev.com/p/32069-In Vitro Cardiovascular Methods. https://sysrev.com/p/33691-In Vitro Cardiovascular Methods-Predictions Subset. 


\section{Description}

Cardiotoxicity is a major cause of failure of new drugs in mid-to late-stage development, cardiovascular disease is one of the leading causes of mortality in the US and worldwide. While cardiovascular health effects of several environmental exposures such as smoking, air pollution, and lead are well characterized, exposure to other environmental chemicals represents another potentially significant but underappreciated risk factor contributing to the development and severity of cardiovascular disease, and reliable methods to detect potential chemical cardiotoxicity need to be identified and applied. Most cardiotoxicity testing is currently done in animals, but these tests are expensive, time-consuming, and can fail to fully predict effects in humans. NICEATM and NTP staff used Sysrev.com to conduct literature searches to identify in vitro methods with mechanistic targets that can be mapped to one of six identified cardiotoxicity "failure modes" (Krishna et al., 2020). These methods could be used to develop integrated testing strategies that are predictive of cardiovascular toxicity and provide human-relevant mechanistic information.

\section{Funding}

This project was funded with federal funds from the NIEHS, NIH under Contract No. HHSN273201500010C.

\section{ADDITIONAL PROJECTS.}

We are grateful to the many teams that submitted case studies. There have now been thousands of public projects on Sysrev, and we were unable to request case studies from all project owners. Other projects can be discovered by using the Sysrev. com search functionality. Sysrev indexes all public projects and makes them discoverable by major search engines and via the on-site search function. For example, to find projects, users, and groups related to "cancer" use the search https://sysrev. $\mathrm{com} /$ search? $\mathrm{q}=$ cancer\&p=1\&type=projects. This results in 35 projects.

\section{META-ANALYSIS}

Sysrev generates millions of data points for article labels, model predictions, and projects. Sysrev actively uses that data to improve the visibility of systematic review, build a review community, and improve the state of machine learning for human evidence review and data extraction.

\section{A Review Community}

As of March 2021, 3,224 sysrevs have been created on Sysrev.com, 442,265 documents have been reviewed, and 1.8 million review tasks have been completed. Sysrev aims to provide a platform where reviewer communities can easily form to develop and share their methods and data.

The force layout diagram in Figure 6A looks at 3 of the larger communities on sysrev.com. Each pink node is a user, and each green node is a sysrev. Connections are drawn between sysrevs and their members. Each of these authors and a selection of their projects are referenced in the case studies section.

Grames and Keenan were recipients of Sysrev mini-grants, which helped in small part to fund their research on insect population biodiversity trends (see case study) and chronic kidney disease in the context of Covid-19 (see case study). Both authors collaborated with Dr. Haddaway (referenced in several case studies), and altogether the three have spawned multiple reviewer communities.

Bozada is a sysrev.com employee who has created many projects (public and private) for managed reviews. There is a chain of reviewer/sysrev membership links that lead to sysrev. $\mathrm{com} / \mathrm{p} / 3588$ and sysrev.com $/ \mathrm{p} / 100$ which are projects completed by toxicology and cancer research groups, the latter of which represents a hepatotoxicity project by the Evidence-Based Toxicology Collaboration led by Dr. Tsaioun at Johns Hopkins which provided support for a report on "Performance of preclinical models in predicting drug-induced liver injury in humans: a systematic review" (Dirven et al., 2021).

Figure 6B Shows the progression of public project sysrev.com/ $\mathrm{p} / 3509$. This is an educational project teaching students to review toxicology data. It is an assignment of a coursera course on evidence based toxicology by Dr. Smirnova at Johns Hopkins School of Public Health. As of March 1st, 2021, over 170 students have extracted 24,791 label answers from 1,581 zebrafish toxicology documents, making this one of Sysrev's largest public projects by number of users.

\section{Active Learning to Accelerate Review}

Sysrev uses active learning to train screening algorithms and prioritize reviews. The method Sysrev uses is to first distribute random articles for review, then train a model on reviewer answers, then distribute articles with the highest prediction entropy for inclusion (closest to 0.5 probability for inclusion), then retrain models, and repeat the process. The intention of active learning is to accelerate machine learning, so as to build accurate models as quickly as possible and minimize the use of reviewer times on easily modeled documents.

Figure $7 \mathrm{~A}$ evaluates the normalized log score of models as a function of the number of articles that are reviewed before, and are subsequently used in model training. The normalized $\log$ score is calculated on the last $20 \%$ of reviewed articles (articles that have not been used in the training set for any of the visualized models). 64 projects were chosen for this analysis that had at least 1,000 reviewed articles, and had a minimum of $20 \%$ included articles and $20 \%$ excluded articles. This was done because Sysrev model accuracy and balanced accuracy suffers in projects with a highly imbalanced inclusion/exclusion ratio. Articles that were discordant (receiving an include from one reviewer and an exclude from a different reviewer) were discarded. The formula used for normalized score was:

$$
\frac{\log \_s c o r e-m_{i n} \log \_s c o r e}{\text { max_log_score }- \text { min_log_score }}
$$



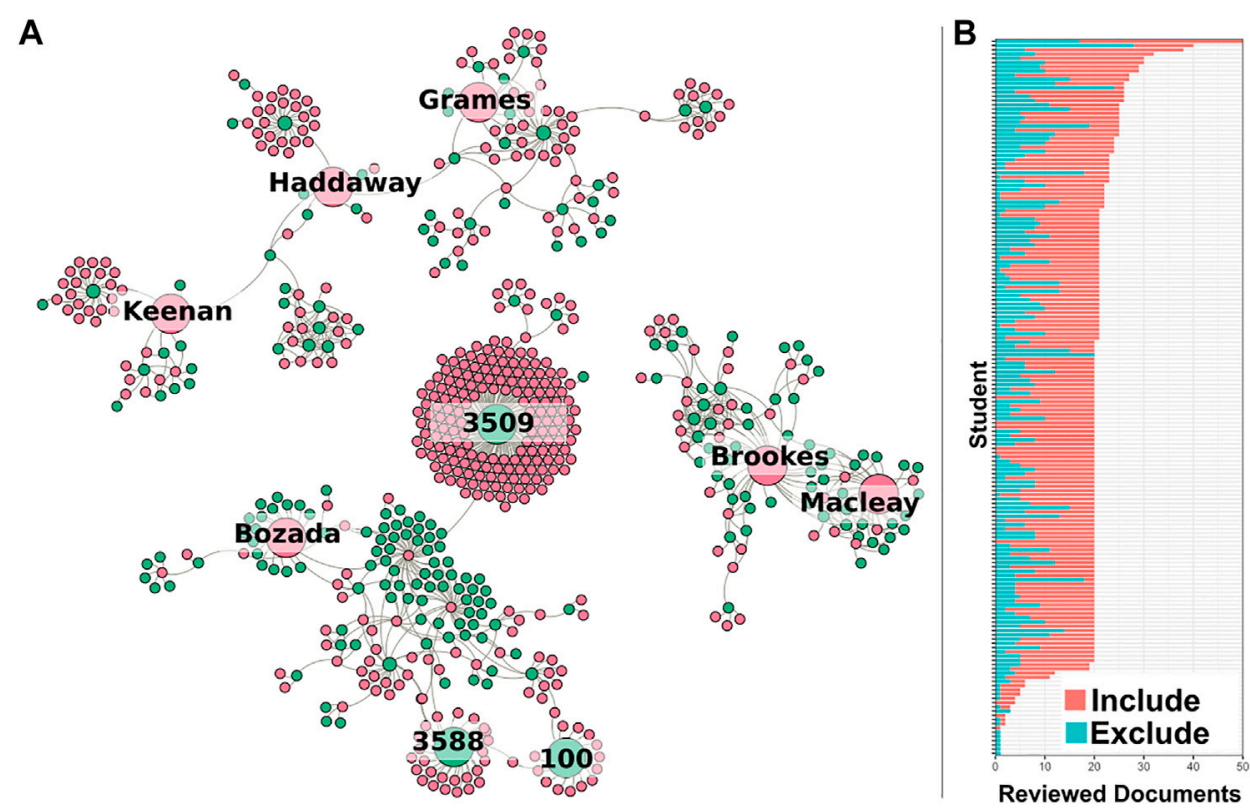

FIGURE 6 | (A) Three of the larger communities on sysrev.com. Pink nodes represent users, green nodes represent projects. Edges link projects with their users. Several notable projects and users have been enlarged for discussion. (B) Bar chart representing completed document reviews for each student in sysrev.com/p/3509, an educational review focused on evidence based toxicology by Dr. Lena Smirnova. Each bar represents a student, students were asked to complete a review of 20 documents, blue bars indicate excluded articles, red bars indicate included articles in an article screening exercise.

Log score is the logarithmic scoring rule for evaluating prediction models, this is a proper scoring rule that assigns greater values to models that assign high confidence to correct predictions, and low confidence to incorrect predictions (Bickel, 2007). In this case, log score is maximized (with a value of 0 ) by models that assign a 1.0 prediction to articles that were concordantly reviewed as inclusion articles and a 0.0 prediction to articles that were concordantly excluded. An additional normalization method of subtracting the log score of the worst performing model in a project and dividing by the difference between the log score of the best and worst model in a project transforms this metric into a proportion receiving a value of 0.0 for the worst models in a project and a value of 1.0 for the best. Values closer to 1.0 indicate model performance close to the best achieved in a project. This normalization method is used to make models with different baseline performance more comparable and allow for an aggregate evaluation of the number of articles required to build models close to maximum performance in a sysrev.

Figure 7A provides a boxplots visualizing inclusion model performance relative to the best and worst inclusion model for a project, a value of $100 \%$ indicates that the model has log score performance equal to the best model for a project, $0 \%$ indicates a model with log score equal to the worst model for the project. The median performance (relative to the best project model) of the first models built in projects (after a review of just 30 articles) is close to $0 \%$ for most projects. After reviewing 690 articles, most models are relatively close to the best model that will ever be achieved for their projects. There is no guarantee that models will perform well after reviewing 690 articles, actual performance is dependent on many factors. This figure simply demonstrates that the Sysrev model generating algorithm usually converges to some maximum performance for the average sysrev after a few hundred articles have been reviewed. In some cases this convergence occurs much earlier, and in some cases it occurs much later. One of the goals of active learning is to accelerate the pace at which maximum model performance is achieved.

Inclusion model performance metrics are visualized as a function of articles reviewed before model training for the entogem sysrev (see case study and sysrev.com/p/16612) in Figure 7B. Dr. Grames and her team reviewed 7,091 articles, the last $20 \%$ of articles were used to evaluate models, $\sim 1,500$ discordant articles were not included in this analysis. Model accuracy reaches a maximum after $\sim 1,000$ concordant articles have been reviewed, model balanced accuracy actually declines slightly to $82.5 \%$ at the 1,000 article point. Log score continues to improve for several thousand more article reviews. This indicates that, for this project, the models continue to improve by generating higher confidence correct predictions and lower confidence incorrect predictions, with only small changes in the total number of incorrect and correct predictions. Models with high confidence of correct predictions, and low confidence of incorrect predictions are more valuable than otherwise equally accurate models.

While proper scoring rules like log score provide a good means for comparing model performance, sometimes it is easier to interpret accuracy (number of correct predictions divided by total predictions) and balanced accuracy (an average of sensitivity 

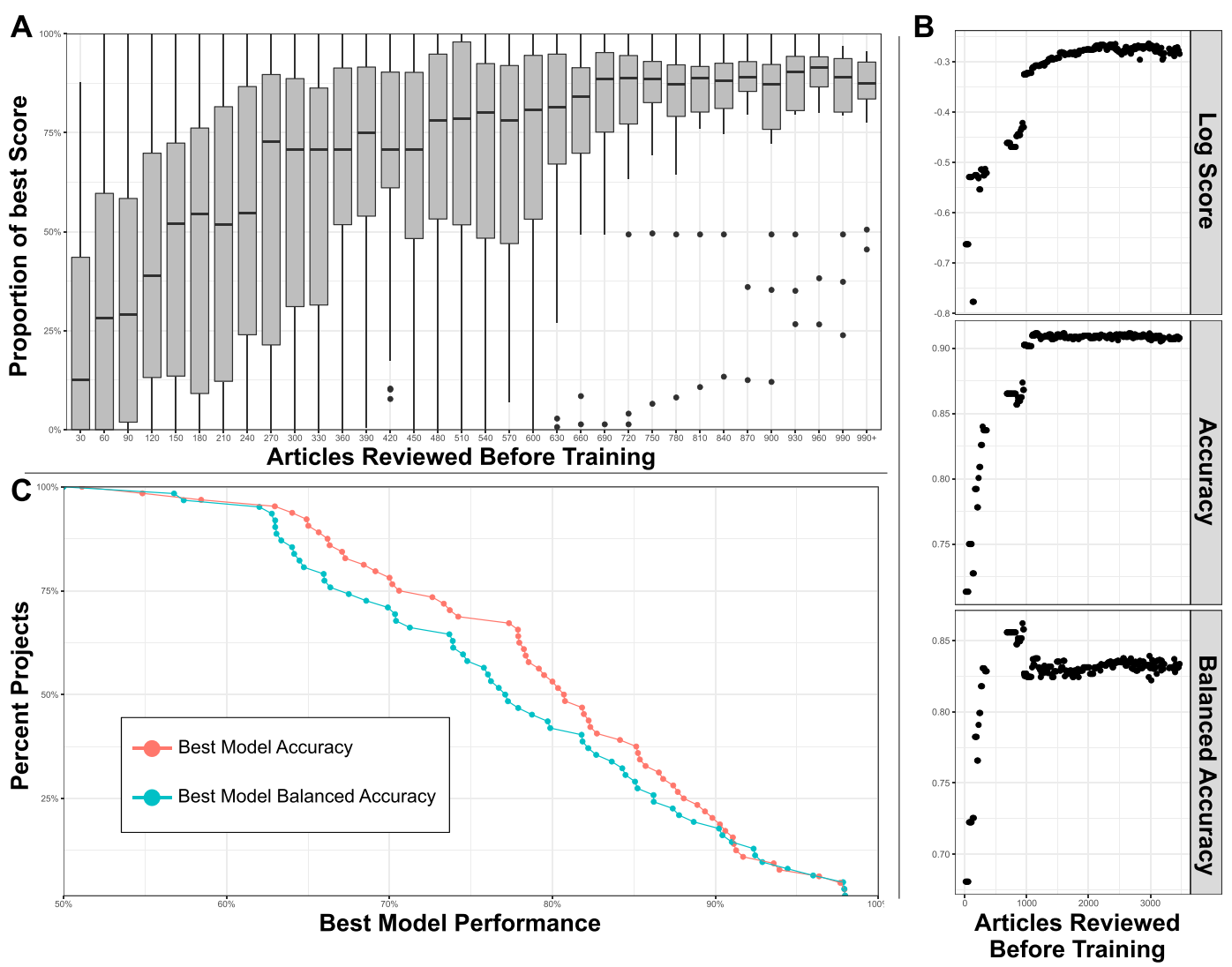

FIGURE 7 | (A) Box plots indicate distribution of model performance relative to the worst and best model in a given model's project. Models are bucketed according to the number of articles labeled before model training. Models improve rapidly until 300 articles have been reviewed. (B) Accuracy metrics for a large sysrev reviewing insect population changes. Model performance is charted as a function of number of articles used in training, across 3 performance metrics, and evaluated on a consistent holdout set. (C) Best model accuracy and balanced accuracy evaluated in 64 sysrevs.

and specificity, correctly predicted inclusions and exclusions in this case). Figure 7C provides a cumulative distribution of the best model accuracy and best model balanced accuracy aggregated over projects. Of the 64 surveyed projects, $75 \%$ had a balanced accuracy and accuracy of $68 \%$ or higher, $50 \%$ of these projects accurately predicted concordant human screening decisions $80 \%$ of the time or higher. None of the models achieved perfect performance. It is our belief (untested in this paper), that Sysrev screening model performance is strongly dependent on the types of articles being reviewed, on the reviewers performing the project, on the complexity of inclusion criteria, on statistical factors like the balance of included articles to excluded articles and many other factors. There is no guarantee that Sysrev models will perform well on a given project and Sysrev provides tools to evaluate model performance during project progression to allow administrators to determine the value of these tools on a project by project basis. Nevertheless, it is informative to observe that, in aggregate, these models have performed well for many sysrevs. This is a difficult task that indicates that the sysrev model architecture and training method generalizes well across many different kinds of projects. Future research will provide more information on sysrev modeling efforts.

\section{CONCLUSION}

We developed Sysrev to be a FAIR-compliant, discipline-agnostic platform for data curation and SERs. Sysrev can be easily adopted for non-biomedical research, such as environmental, climate, or industrial purposes. Existing datasets are often proprietary or have limited applicability beyond their original purpose, which contributes to the promulgation of data silos. Importantly, much of the data captured in both primary research and SERs is published in a manner that inhibits the application of the data to be re-used for new investigations. This is especially problematic for investigations where users may not be aware of relevant data from cross-discipline/industry studies. Sysrev's public projects are accessible, clonable, and transparent to encourage the responsible reuse of data and support broad research goals.

There are a growing number of tools in the document review and systematic review space. The Systematic Review Toolbox provides a strong resource for quickly finding and comparing other tools to Sysrev (Marshall and Sutton, 2021). The pace of platform development makes it difficult to compare feature development, however, Dr. Leenaars provides an introduction to several tools in her publication "Software tools for literature 
screening in systematic reviews in biomedical research." (van der Mierden et al., 2019).

Sysrev has been used for thousands of review and data curation projects. Users agree that Sysrev makes the SER process run more efficiently, even with large teams of more than 100 users or those with tens of thousands of studies/data. Further improvements to improve the user experience are planned and in development. Sysrev offers several access options, including a free version with unlimited publicly available projects or pro/enterprise versions that include additional functionality. Sysrev is privately funded by insilica. co and can be accessed at https://sysrev.com.

\section{DATA AVAILABILITY STATEMENT}

The datasets presented in this study can be found in online repositories. The names of the repository/repositories and accession number(s) can be found below: sysrev.com.

\section{REFERENCES}

Arnaud, E., Laporte, M.-A., Kim, S., Aubert, C., Leonelli, S., Miro, B., et al. (2020). The Ontologies Community of Practice: A CGIAR Initiative for Big Data in Agrifood Systems. Patterns 1, 100105. doi:10.1016/j.patter.2020.100105

Bickel, J. E. (2007). Some Comparisons Among Quadratic, Spherical, and Logarithmic Scoring Rules. Decis. Anal. 4, 49-65. doi:10.1287/deca.1070.0089

Bilynsky, C., Han, W., Gupta, A., Aldarondo, D., Fox, H., Brown, C., et al. (2021). Scoping Review of Pre-clinical and Translational Studies on Macrophage Polarization in Nanoparticle-Based Cancer Immunotherapy. doi:10.17605/ OSF.IO/HWD2R

Bozada, T. (2020). What Is Sysrev for? Literature Reviews and Data Curation. Available at: https://blog.sysrev.com/literature-review-data-curation/.

Bozada, T. J. (2020). Supporting COVID Research: Rapid Reviews on Sysrev. Available at: https://blog.sysrev.com/covid-rapid-review/ (Accessed Mar 16, 2021).

Burns, P. B., Rohrich, R. J., and Chung, K. C. (2011). The Levels of Evidence and Their Role in Evidence-Based Medicine. Plast. Reconstr. Surg. 128, 305-310. doi:10.1097/prs.0b013e318219c171

Cáceres-Escobar, H., Broad, S., Challender, D. W. S., Kinnaird, M. F., Keenan, C., Lichtenstein, G., et al. (2020). PROTOCOL: An Evidence and Gap Map (EGM) about the Roles and Risks of Wildife Trade in the Emergence of Human Infectious Diseases and Zoonoses. doi:10.6084/m9.figshare.13392275.v2

Daszak, P., das, Neves. C., Amuasi, J., Haymen, D., Kuiken, T., Roche, B., et al. (2020). Workshop Report on Biodiversity and Pandemics of the Intergovernmental Platform on Biodiversity and Ecosystem Services. Bonn, Germany: IPBES secretariat.

David, E., Madec, S., Sadeghi-Tehran, P., Aasen, H., Zheng, B., Liu, S., et al. (2020). Global Wheat Head Detection (GWHD) Dataset: A Large and Diverse Dataset of High-Resolution RGB-Labelled Images to Develop and Benchmark Wheat Head Detection Methods. Plant Phenomics 2020, 1-12. doi:10.34133/2020/ 3521852

Dirven, H., Vist, G. E., Bandhakavi, S., Mehta, J., Fitch, S. E., Pound, P., et al. (2021). Performance of Preclinical Models in Predicting Drug-Induced Liver Injury in Humans: a Systematic Review. Sci. Rep. 11, 6403. doi:10.1038/s41598-021-85708-2

Gates, A., Guitard, S., Pillay, J., Elliott, S. A., Dyson, M. P., Newton, A. S., et al. (2019). Performance and Usability of Machine Learning for Screening in Systematic Reviews: a Comparative Evaluation of Three Tools. Syst. Rev. 8, 278. doi:10.1186/s13643-019-1222-2

Grames, E., Montgomery, G. A., Haddaway, N. R., Dicks, L. V., Elphick, C. S., Matson, T. A., et al. (2019). Trends in Global Insect Abundance and Biodiversity: A Community-Driven Systematic Map Protocol. doi:10.17605/osf.io/q63uy

Grames, E. (2020). Preliminary Results from the EntoGEM Project May 06, 2020. doi:10.5281/zenodo.3814732

\section{AUTHOR CONTRIBUTIONS}

TB is the director of managed reviews at sysrev.com where he helps users and institutions to get the most out of the platform. JB is a developer at sysrev.com, specializing in the construction of data sources. JW is the first developer at sysrev.com and architect of the technical infrastructure. MD is a new developer at sysrev.com, and involved in full stack development of the service. JM is an expert in evidence based medicine, and provided help writing and communicating with cause studies. TL is the founder of sysrev.com and a contributor in all aspects of the project.

\section{FUNDING}

This project is funded by Insilica, LLC.

Hariri, R. H., Fredericks, E. M., and Bowers, K. M. (2019). Uncertainty in Big Data Analytics: Survey, Opportunities, and Challenges. J. Big Data 6, 44. doi:10.1186/ s40537-019-0206-3

Harrison, H., Griffin, S. J., Kuhn, I., and Usher-Smith, J. A. (2020). Software Tools to Support Title and Abstract Screening for Systematic Reviews in Healthcare: an Evaluation. Bmc Med. Res. Methodol. 20, 7. doi:10.1186/ s12874-020-0897-3

Kelley, G. A., and Kelley, K. S. (2018). Systematic Reviews and Cancer Research: a Suggested Stepwise Approach. Bmc Cancer 18, 246. doi:10.1186/s12885-0184163-6

Kilgore, W. W. (1990). California's Proposition 65: Extrapolating Animal Toxicity to Humans. Am. J. Ind. Med. 18, 491-494. doi:10.1002/ajim.4700180422

Krishna, S., Berridge, B., and Kleinstreuer, N. (2020). High-Throughput Screening to Identify Chemical Cardiotoxic Potential. Chem. Res. Toxicol. 34, 566-583. doi:10.1021/acs.chemrestox.0c00382

Luechtefeld, T., and Bozada, T. (2019). How Does Machine Learning Work at Sysrev.Com. Available at: https://blog.sysrev.com/machine-learning.

Luechtefeld, T. (2019). Sysrev Helps Create Chemical Transparency for Manufacturers. Available at: https://blog.sysrev.com/srg-sysrev-chemicaltransparency/ (Accessed Mar 16, 2021).

Mahalingasivam, V., Craik, A., Tomlinson, L. A., Ge, L., Hou, L., Wang, Q., et al. (2021). A Systematic Review of COVID-19 and Kidney Transplantation. Kidney Int. Rep. 6, 24-45. doi:10.1016/j.ekir.2020.10.023

Mallett, R., Hagen-Zanker, J., Slater, R., and Duvendack, M. (2012). The Benefits and Challenges of Using Systematic Reviews in International Development Research. J. Dev. Effectiveness 4, 445-455. doi:10.1080/19439342.2012.711342

Marshall, C., and Sutton, A. (2021). Systematic Review Toolbox. Available at: http://systematicreviewtools.com/about.php (Accessed Jun 17, 2021).

Muhamad, N., Plengsuriyakarn, T., and Na-Bangchang, K. (2018). Application of Active Targeting Nanoparticle Delivery System for Chemotherapeutic Drugs and Traditional/herbal Medicines in Cancer Therapy: a Systematic Review. Ijn 13, 3921-3935. doi:10.2147/ijn.s165210

Noy, R., and Pollard, J. W. (2014). Tumor-Associated Macrophages: From Mechanisms to Therapy. Immunity 41, 49-61. doi:10.1016/j.immuni.2014.06.010

Paller, C., Tamashiro, T., Luechtefeld, T., Gravell, A., and Levine, M. (2020). "Vitamin C and Cancer an Overview of Recent Clinical Trials," in Vissers QC and MCMCancer and Vitamin C. 1st Edition, 64.

P. Bryan Heidorn, P. B. (2008). Shedding Light on the Dark Data in the Long Tail of Science. Libr. Trends 57, 280-299. doi:10.1353/lib.0.0036

Plasterer, T., Romacker, A., Barron, R., and Harrow, B. (2021). FAIR Toolkit - the FAIR Toolkit by Pistoia Alliance - A FAIR Toolkit for Life Science Industry. Available at: https://fairtoolkit.pistoiaalliance.org/ (Accessed Jun 17, 2021).

Salvioni, L., Rizzuto, M. A., Bertolini, J. A., Pandolfi, L., Colombo, M., and Prosperi, D. (2019). Thirty Years of Cancer Nanomedicine: Success, Frustration, and Hope. Cancers 11, 1855. doi:10.3390/cancers11121855 
Saunders, M. E., Barton, P. S., Bickerstaff, J. R. M., Frost, L., Latty, T., Lessard, B. D., et al. (2021). Limited Understanding of Bushfire Impacts on Australian Invertebrates. Insect Conserv Divers., 14, 285, 293. doi:10.1111/icad.12493

Settles, B. (2009). Active Learning Literature Survey Madison, WI, United States: University of Wisconsin-Madison, Department of Computer Sciences.

Sterne, J. A., Hernán, M. A., Reeves, B. C., Savović, J., Berkman, N. D., Viswanathan, M., et al. (2016). ROBINS-I: a Tool for Assessing Risk of Bias in Non-randomised Studies of Interventions. Bmj 355, i4919. doi:10.1136/ bmj.i4919

Tryka, K. A., Hao, L., Sturcke, A., Jin, Y., Wang, Z. Y., Ziyabari, L., et al. (2014). NCBI's Database of Genotypes and Phenotypes: dbGaP. Nucl. Acids Res. 42, D975-D979. doi:10.1093/nar/gkt1211

UNEP, ILRI (2020). Preventing the Next Pandemic: Zoonotic Diseases and How to Break the Chain of Transmission.

van der Mierden, S., Tsaioun, K., Bleich, A., and Leenaars, C. H. C. (2019). Software Tools for Literature Screening in Systematic Reviews in Biomedical Research. Altex 36, 508-517. doi:10.14573/altex.1902131

Wallis, J. C., Rolando, E., and Borgman, C. L. (2013). If We Share Data, Will Anyone Use Them? Data Sharing and Reuse in the Long Tail of Science and Technology. Plos One 8, e67332. doi:10.1371/ journal.pone.0067332

Wilkinson, M. D., Dumontier, M., Aalbersberg, I. J., Appleton, G., Axton, M., Baak, A., et al. (2016). The FAIR Guiding Principles for Scientific Data Management and Stewardship. Sci. Data 3, 160018. doi:10.1038/ sdata.2016.18

Wise, J., de Barron, A. G., Splendiani, A., Balali-Mood, B., Vasant, D., Little, E., et al. (2019). Implementation and Relevance of FAIR Data Principles in Biopharmaceutical R\&D. Drug Discov. Today 24, 933-938. doi:10.1016/ j.drudis.2019.01.008
Yang, B., Sun, J.-T., Wang, T., and Chen, Z. (2009). "Effective Multi-Label Active Learning for Text Classification," in KDD '09: Proceedings of the 15th ACM SIGKDD international conference on Knowledge discovery and data mining, 28 June 2009 (New York, NY, United States: ACM), 917-926. doi:10.1145/ 1557019.1557119

Zhou, J., Tang, Z., Gao, S., Li, C., Feng, Y., and Zhou, X. (2020). Tumor-Associated Macrophages: Recent Insights and Therapies. Front. Oncol. 10, 188. doi:10.3389/fonc. 2020.00188

Conflict of Interest: TB, JB, JW, MD, and TL was employed by Insilica LLC. TL was employed by Toxtrack LLC.

The remaining authors declare that the research was conducted in the absence of any commercial or financial relationships that could be construed as a potential conflict of interest.

Publisher's Note: All claims expressed in this article are solely those of the authors and do not necessarily represent those of their affiliated organizations, or those of the publisher, the editors and the reviewers. Any product that may be evaluated in this article, or claim that may be made by its manufacturer, is not guaranteed or endorsed by the publisher.

Copyright (C) 2021 Bozada, Borden, Workman, Del Cid, Malinowski and Luechtefeld. This is an open-access article distributed under the terms of the Creative Commons Attribution License (CC BY). The use, distribution or reproduction in other forums is permitted, provided the original author(s) and the copyright owner(s) are credited and that the original publication in this journal is cited, in accordance with accepted academic practice. No use, distribution or reproduction is permitted which does not comply with these terms. 